\title{
DOMAIN DECOMPOSITION FOR LESS REGULAR SUBDOMAINS: OVERLAPPING SCHWARZ IN TWO DIMENSIONS*
}

\author{
CLARK R. DOHRMANN ${ }^{\dagger}$, AXEL KLAWONN ${ }^{\ddagger}$, AND OLOF B. WIDLUND $^{\S}$
}

\begin{abstract}
In the theory of domain decomposition methods, it is often assumed that each subdomain is the union of a small set of coarse triangles or tetrahedra. In this study, extensions to the existing theory which accommodate subdomains with much less regular shapes are presented; the subdomains are required only to be John domains. Attention is focused on overlapping Schwarz preconditioners for problems in two dimensions with a coarse space component of the preconditioner, which allows for good results even for coefficients which vary considerably. It is shown that the condition number of the domain decomposition method is bounded by $C(1+H / \delta)(1+\log (H / h))^{2}$, where the constant $C$ is independent of the number of subdomains and possible jumps in coefficients between subdomains. Numerical examples are provided which confirm the theory and demonstrate very good performance of the method for a variety of subregions including those obtained when a mesh partitioner is used for the domain decomposition.
\end{abstract}

Key words. domain decomposition, overlapping Schwarz, preconditioners, iterative methods, irregular subdomains, John domains

AMS subject classifications. 65F10, 65N30, 65N55

DOI. $10.1137 / 070685841$

1. Introduction. In the theory for overlapping Schwarz domain decomposition methods, we typically assume that each subdomain is quite regular, e.g., the union of a small set of coarse triangles or tetrahedra; see, e.g., [28, Assumption 4.3]. However, this is often unrealistic, especially if the subdomains result from using a mesh partitioner. The subdomain boundaries might then not even be uniformly Lipschitz continuous; i.e., the number of patches required to cover the boundary of the region, in each of which the boundary is the graph of a Lipschitz continuous function, might not be uniformly bounded independently of the finite element mesh size. We note that the existing theory for iterative substructuring establishes bounds on the convergence rates of the algorithms which are insensitive to even large jumps in the material properties across subdomain boundaries as reflected in the coefficients of the problem. The theory for overlapping Schwarz methods is less restrictive as far as the subdomain shapes are concerned (see, e.g., [28, Chapter 3]) but relatively little has been known about the effect of large changes in the coefficients; see, however, [25] and recent work reported in $[13,26]$.

The purpose of this paper is to begin the development of a theory under much weaker assumptions on the partitioning; a companion study for iterative substruc-

* Received by the editors March 21, 2007; accepted for publication (in revised form) December 17, 2007; published electronically May 2, 2008.

http://www.siam.org/journals/sinum/46-4/68584.html

†Structural Dynamics Research Department, Sandia National Laboratories, Albuquerque, NM 87185 (crdohrm@sandia.gov). Sandia is a multiprogram laboratory operated by Sandia Corporation, a Lockheed Martin Company, for the United States Department of Energy's National Nuclear Security Administration under contract DE-AC04-94AL85000.

${ }^{\ddagger}$ Fachbereich Mathematik, Universität Duisburg-Essen, Universitätsstr. 3, 45117 Essen, Germany (axel.klawonn@uni-due.de).

$\S$ Department of Mathematics, Courant Institute, New York, NY 10012 (widlund@cims.nyu.edu). This author's work was supported in part by the U.S. Department of Energy under contracts DEFG02-06ER25718 and DE-FC02-01ER25482 and in part by National Science Foundation grant DMS0513251. 
turing methods, by Rheinbach and the second and third authors of this paper, has also been completed [16]. Our results in this paper concern an overlapping Schwarz algorithm which combines a coarse space adopted from an iterative substructuring method (see [28, Algorithm 5.16] and [11]) with local preconditioner components selected as in classical overlapping Schwarz methods, i.e., based on solving problems on overlapping subdomains. This choice of the coarse component will allow us to prove results which are independent of coefficient jumps. We note that there are earlier studies on multilevel methods $[10,24]$ in which the coarsest components are similarly borrowed from iterative substructuring algorithms.

We will use nonoverlapping subdomains and denote them by $\Omega_{i}, i=1, \ldots, N$, as well as overlapping subdomains $\Omega_{j}^{\prime}, j=1, \ldots, N^{\prime}$. The interface between the $\Omega_{i}$ will be denoted by $\Gamma$.

So far, complete results have been obtained only for problems in the plane. To simplify our presentation, we will also confine ourselves to scalar elliptic problems of the following form:

$$
-\nabla \cdot(\rho(x) \nabla u(x))=f(x), \quad x \in \Omega \subset \mathbb{R}^{2},
$$

with a Dirichlet boundary condition on a measurable subset $\partial \Omega_{D}$ of $\partial \Omega$, the boundary of $\Omega$, and a Neumann condition on $\partial \Omega_{N}=\partial \Omega \backslash \partial \Omega_{D}$. The coefficient $\rho(x)$ is strictly positive and assumed to be a constant $\rho_{i}$ for $x \in \Omega_{i}$. We use piecewise linear, continuous finite elements and triangulations with shape-regular elements and assume that each subdomain is the union of a set of elements. The weak formulation of the elliptic problem is written in terms of the bilinear form

$$
a(u, v):=\sum_{i=1}^{N} a_{i}(u, v):=\sum_{i=1}^{N} \rho_{i} \int_{\Omega_{i}} \nabla u \cdot \nabla v d x .
$$

Our study requires the generalization of some technical tools used in the proof of a bound of the convergence rate of this type of algorithm. Some of the standard tools, such as those collected in [28, section 4.6], are no longer available, and we therefore also have to modify the reasoning in the proof of our main result. Three auxiliary results, namely, a Poincaré inequality, a Sobolev-type inequality for finite element functions, and a bound for certain edge terms, will be required in our proof; see Lemmas 2.2, 3.2, and 3.4. We will work with John domains, introduced in section 2 , and will be able to express our bounds on the convergence of our algorithm in terms of a few geometric parameters.

2. A Poincaré inequality and John domains. In any analysis of any domain decomposition method with more than one level, we need a Poincaré inequality. This inequality is closely related to an isoperimetric inequality; see [12, 20, 18, 21, 22].

Lemma 2.1 (isoperimetric inequality). Let $\Omega \subset \mathbb{R}^{n}$ be a domain-an open, bounded, and connected set-and let $u$ be sufficiently smooth. Then

$$
\inf _{c \in R}\left(\int_{\Omega}|u-c|^{n /(n-1)} d x\right)^{(n-1) / n} \leq \gamma(\Omega, n) \int_{\Omega}|\nabla u| d x
$$

if and only if

$$
(\min (|A|,|B|))^{1-1 / n} \leq \gamma(\Omega, n)|\partial A \cap \partial B| .
$$

Here $A \subset \Omega$ is an arbitrary open set, and $B=\Omega \backslash \bar{A} ; \gamma(\Omega, n)$ is the best possible constant, and $|A|$ is the measure of the set $A$, etc. 
We note that the domain does not need to be star-shaped or Lipschitz. A small value of $\gamma(\Omega, n)$ is desirable for our purposes. Thus, the domain cannot be very thin, and its boundary should not have very pointed parts.

For two dimensions, we immediately obtain a standard Poincaré inequality by using the Cauchy-Schwarz inequality. The best choice of $c$ is $\bar{u}_{\Omega}$, the average of $u$ over the domain.

Lemma 2.2 (Poincaré's inequality). Consider a domain $\Omega \subset \mathbb{R}^{2}$. Then

$$
\left\|u-\bar{u}_{\Omega}\right\|_{L_{2}(\Omega)}^{2} \leq(\gamma(\Omega, 2))^{2}|\Omega|\|\nabla u\|_{L_{2}(\Omega)}^{2} \quad \forall u \in H^{1}(\Omega) .
$$

For $n=3$, a similar bound is obtained by using Hölder's inequality several times. In Lemma 2.2 , we then should replace $|\Omega|$ by $|\Omega|^{2 / 3}$.

Throughout, we will use weighted $H^{1}\left(\Omega_{i}\right)$-norms defined by

$$
\|u\|_{H^{1}\left(\Omega_{i}\right)}^{2}:=|u|_{H^{1}\left(\Omega_{i}\right)}^{2}+1 / H_{i}^{2}\|u\|_{L_{2}\left(\Omega_{i}\right)}^{2}:=\int_{\Omega_{i}} \nabla u \cdot \nabla u d x+1 / H_{i}^{2} \int_{\Omega_{i}}|u|^{2} d x .
$$

Here $H_{i}$ is the diameter of $\Omega_{i}$. The weight originates from a dilation of a domain with diameter 1 . We will use Lemma 2.2 to remove $L_{2}$-terms from full $H^{1}$-norms.

We will also use another variant of the Poincaré inequality; in fact we will use it only in the simple case when the region is a circular disk.

Lemma 2.3 (Poincaré, $L_{1}$-variant). Consider a domain $\Omega \subset \mathbb{R}^{2}$. Then

$$
\left\|u-\bar{u}_{\Omega}\right\|_{L_{1}(\Omega)} \leq \gamma(\Omega, 2)|\Omega|^{1 / 2}\|\nabla u\|_{L_{1}(\Omega)} \quad \forall u \in W^{1,1}(\Omega) .
$$

Proof. By the Cauchy-Schwarz inequality,

$$
\left\|u-\bar{u}_{\Omega}\right\|_{L_{1}(\Omega)} \leq|\Omega|^{1 / 2}\left\|u-\bar{u}_{\Omega}\right\|_{L_{2}(\Omega)} .
$$

The proof is then completed by using Lemma 2.1.

We next give a definition of a John domain; see [14] and the references therein.

DEFINITION 2.4 (John domain). A domain $\Omega \subset \mathbb{R}^{n}$ is a John domain if there exists a constant $C_{J} \geq 1$ and a distinguished central point $x_{0} \in \Omega$ such that each $x \in \Omega$ can be joined to it by a rectifiable curve $\gamma:[0,1] \rightarrow \Omega$ such that $\gamma(0)=x_{0}, \gamma(1)=x$, and distance $(\gamma(t), \partial \Omega) \geq C_{J}^{-1}|x-\gamma(t)|$ for all $t \in[0,1]$.

This condition can be viewed as a twisted cone condition. We note that certain snowflake curves with fractal boundaries are John domains and that the length of the boundary of a John domain can be arbitrarily much larger than its diameter; see Figure 5.1. We also note that, by restricting the refinement to the neighborhood of a point, the contribution to the length of the boundary from a small neighborhood of the point can be made to dominate. A John domain can have cusps facing inwards but not outwards; this means, however, that there can be no cusps on the interface since any cusp would face outwards for one of two neighboring subdomains; see [21, 22] for further information.

We note that, for any choice of the point $x_{0}$, there is a point $x \in \Omega$ at a distance of at least diameter $(\Omega) / 2$. We find that diameter $(\Omega) \leq 2 C_{J} r_{\Omega}$, where $r_{\Omega}$ is the radius of the largest ball inscribed in $\Omega$ and centered at $x_{0}$.

It is known that any simply connected plane domain with a finite Poincaré parameter $\gamma(\Omega, 2)$ is a John domain; see [7]. It is also known (see [2]) that any John domain has a bounded Poincaré parameter $\gamma(\Omega, n)$. Since we have been unable to find a simple estimate of $\gamma(\Omega, n)$ in terms of $C_{J}$ in the literature, we have chosen to express our results in terms of both of these geometric parameters.

Copyright $@$ by SIAM. Unauthorized reproduction of this article is prohibited. 
3. The algorithm, technical tools, and the main result. The domain $\Omega \subset$ $\mathbb{R}^{2}$ of the elliptic problem is decomposed into nonoverlapping subdomains $\Omega_{i}$, each of which is the union of elements, and with the finite element nodes on the boundaries of neighboring subdomains matching across the interface $\Gamma$, which is the union of the parts of the subdomain boundaries which are common to at least two subdomains. The interface $\Gamma$ is composed of edges and vertices. An edge $\mathcal{E}^{i k}$ is an open subset of $\Gamma$, which contains the nodes which are shared only by the boundaries of a particular pair of subdomains $\Omega_{i}$ and $\Omega_{k}$. The subdomain vertices $\mathcal{V}^{\ell}$ are end points of edges and are typically shared by more than two; see [17, Definition 3.1] for more details on how these sets can be defined for quite general situations. We denote the standard finite element space of continuous, piecewise linear functions on $\Omega_{i}$ by $V^{h}\left(\Omega_{i}\right)$ and assume that these functions vanish at the nodes on $\partial \Omega_{i} \cap \partial \Omega_{D}$.

We will view our algorithm as an additive Schwarz method, as in [28, Chapters 2 and 3], defined in terms of a set of subspaces. To simplify the discussion, we will use exact solvers for both the coarse problem and the local ones. All that is then required for the analysis of our algorithm is an estimate of a parameter in a stable decomposition of any function in the finite element space; see [28, Assumption 2.2 and Lemma 2.5]. Thus, we need to estimate $C_{0}^{2}$ in

$$
a\left(u_{0}, u_{0}\right)+\sum_{j=1}^{N^{\prime}} a_{\Omega_{j}^{\prime}}\left(u_{j}, u_{j}\right) \leq C_{0}^{2} a(u, u) \quad \forall u \in V^{h},
$$

for some decomposition $\left\{u_{j}\right\}$, such that

$$
u=\sum_{j=0}^{N^{\prime}} R_{j}^{T} u_{j}, \quad u_{j} \in V_{j} .
$$

Here $a_{\Omega_{j}^{\prime}}(u, v)$ is the bilinear form obtained by integrating only over the subdomain $\Omega_{j}^{\prime}$, and $R_{j}^{T}: V_{j} \longrightarrow V^{h}$ is an interpolation operator from the space of the $j$ th subproblem $V_{j}$ into the space $V^{h}$. The local spaces $V_{j}, j=1, \ldots N^{\prime}$, are defined as

$$
V_{j}=V^{h}\left(\Omega_{j}^{\prime}\right) \cap H_{0}^{1}\left(\Omega_{j}^{\prime}\right),
$$

except for subdomains with a boundary which intersects $\partial \Omega_{N}$. In the latter case, the functions of the local space are constrained to vanish only at the nodes of $\partial \Omega_{j}^{\prime} \backslash \partial \Omega_{N}$. This is the same standard choice as in [28, Chapter 3]. We will assume that each $\Omega_{j}^{\prime}$ has a diameter comparable to those of the subdomains $\Omega_{i}$ which it intersects.

Associated with each local space $V_{j}$ is a projection $P_{j}$ defined by

$$
a_{\Omega_{j}^{\prime}}\left(\tilde{P}_{j} u, v\right)=a\left(u, R_{j}^{T} v\right) \quad \forall v \in V_{j} \text {, and } P_{j}=R_{j}^{T} \tilde{P}_{j} .
$$

The coarse space $V_{0}$ is spanned by vertex and edge functions extended as discrete harmonic functions into the interiors of the subdomains $\Omega_{i}$. The discrete harmonic extensions minimize the energy; see [28, section 4.4]. There will be one basis function $\theta_{\mathcal{V}^{\ell}}(x)$ for each subdomain vertex; it is the discrete harmonic extension of the interface values of the standard nodal basis function. For each edge $\mathcal{E}^{i k}$ there is also a basis function $\theta_{\mathcal{E}^{i k}}(x) \in V^{h}$, which equals 1 at all nodes on the edge and vanishes at all other interface nodes. The vertex and edge functions provide a partition of unity on the interface $\Gamma$. We will use a projection $P_{0}$ onto $V_{0}$, which is defined by

$$
a\left(P_{0} u, v\right)=a(u, v) \quad \forall v \in V_{0} .
$$


The additive Schwarz operator, the preconditioned operator used in our iteration, is given by

$$
P_{a d}=\sum_{j=0}^{N^{\prime}} P_{j} .
$$

By using [28, Lemmas 2.5 and 2.10], we find that the condition number $\kappa\left(P_{a d}\right)$ of the additive Schwarz operator can be bounded by $\left(N^{C}+1\right) C_{0}^{2}$, where $N^{C}$ is the minimal number of colors required to color the subdomains $\Omega_{j}^{\prime}$ such that no pair of intersecting subdomains have the same color.

The overlap between the subdomains is characterized by parameters $\delta_{j}$, as in [28, Assumption 3.1]; $\delta_{j}$ is the minimum width of the subset $\Omega_{j, \delta_{j}}$ of $\Omega_{j}^{\prime}$ which is also covered by at least one neighboring overlapping subdomain.

We can now formulate our main result, which is also valid for compressible elasticity with piecewise constant Lamé parameters, with jumps only across the interface, provided that the coarse space is enriched to include all rigid body functions; see [8]. The extension to the case of linear elasticity requires a Korn inequality; see [1, 17], and [28, Chapter 8]. We note that there are also recent results on almost incompressible elasticity discretized by mixed finite elements with discontinuous pressure spaces; see [9].

TheOREM 3.1 (condition number estimate). Let $\Omega \subset \mathbb{R}^{2}$ be partitioned into nonoverlapping subdomains $\Omega_{i}$, which are John domains, each with a shape-regular triangulation. The condition number of our domain decomposition method then satisfies

$$
\kappa\left(P_{a d}\right) \leq C(1+H / \delta)(1+\log (H / h))^{2},
$$

where $C>0$ is a constant which depends only on the John and Poincaré parameters of the subdomains, the number of colors required for the overlapping subdomains, and the shape regularity of the elements.

As in many domain decomposition results, $H / h$ is shorthand for $\max _{i}\left(H_{i} / h_{i}\right)$, where $h_{i}$ is the diameter of the smallest element of $\Omega_{i}$. Similarly, $H / \delta$ is the largest ratio of $H_{i}$ and the smallest of the $\delta_{j}$ of the overlapping subregions that intersect $\Omega_{i}$.

The logarithmic bound of our main result can be improved to a first power if all of the subregions have boundaries which are uniformly Lipschitz. This is true, in particular, if they satisfy [28, Assumption 4.3]. If, in addition, the coefficients do not have any large jumps across the interface and the coarse space is enriched to contain all piecewise linear functions on a coarse mesh as in [28, Assumption 4.3], we can also eliminate the logarithmic factors altogether by using ideas from the proof of $[28$, Theorem 3.13].

To prove Theorem 3.1, we need two auxiliary results, in addition to Poincaré's inequality. The first is a discrete Sobolev inequality.

Lemma 3.2 (discrete Sobolev inequality). Let $\Omega_{i}$ be a John domain in the plane with diameter $H$. Then

$$
\left\|u-\bar{u}_{\Omega_{i}}\right\|_{L_{\infty}\left(\Omega_{i}\right)}^{2} \leq C(1+\log (H / h))|u|_{H^{1}\left(\Omega_{i}\right)}^{2} \quad \forall u \in V^{h}\left(\Omega_{i}\right) .
$$

The constant $C>0$ depends only on the John parameter $C_{J}\left(\Omega_{i}\right)$, the Poincaré parameter $\gamma\left(\Omega_{i}, 2\right)$, and the shape regularity of the elements.

We note that this lemma is well-known in the theory of iterative substructuring methods. Proofs for domains satisfying an interior cone condition are given in [4] and 
in [6, section 4.9]; see also [3]. For a different proof, see [28, Lemma 4.15]. For a proof that this inequality is sharp, see [5].

In preparation for our proof of this result for John domains, we will formulate and prove a lemma which is directly inspired by a paper by Hajłasz [14]. We first introduce a set of open circular disks. We consider a John domain $\Omega_{i}$ with diameter $H$ and with a triangulation using a mesh size $h$. Let $\gamma(t)$ be a John curve from a central point $x_{0} \in \Omega_{i}$ to an arbitrary point $x$. A family of circular disks $B_{i}, i \geq 0$, is introduced as follows: the first disk is $B_{0}:=B\left(x_{0}\right.$, dist $\left.\left(x_{0}, \partial \Omega_{i}\right) / 4\right)$, the open disk centered at $x_{0}$ and with radius $r_{0}=\operatorname{dist}\left(x_{0}, \partial \Omega_{i}\right) / 4$. We will assume that $x$ lies outside $2 B_{0}$, the concentric disk with twice the radius of $B_{0}$; the other case is trivial. The other disks are defined similarly as $B_{i}:=B\left(x_{i}, r_{i}\right)$, centered at $x_{i}$ and with radius $r_{i}:=\left|x-x_{i}\right| / 4 C_{J}, i \geq 1$. All of the $x_{i}$ 's lie on the John curve, and all of the $B_{i}$ 's are clearly subsets of $\Omega_{i}$. Given $B_{i}$, we select $x_{i+1} \in \partial B_{i}$ as the last point of exit of $\gamma(t)$ from $B_{i}$.

Several properties of the $B_{i}$ can now be established.

LEMmA 3.3. There exists a constant $M=M\left(C_{J}\right)$ such that

1. no point in $\Omega_{i}$ belongs to more than $M$ of the $B_{i}$ (finite covering);

2. $\left|B_{i} \cup B_{i+1}\right| \leq M\left|B_{i} \cap B_{i+1}\right|$;

3. $r_{i} \rightarrow 0$ and $x_{i} \rightarrow x$ as $i \rightarrow \infty$;

4. the number of $x_{i}$ that are at a distance larger than $r$ from $x$ can be estimated by $M \log (H / r)$.

Proof. Our proof will rely heavily on arguments developed in [14], in particular, on the proof of Theorem 10 of that paper, which we will reproduce in part; see also references to earlier contributions in that paper.

Let a point $y$ belong to $B_{k}, B_{k+1}, \ldots B_{k+p}$. Then

$$
|x-y| \leq\left|x-x_{k+m}\right|+\left|x_{k+m}-y\right| \leq\left(4 C_{J}+1\right) r_{k+m}, \quad m=0, \ldots, p .
$$

Similarly,

$$
4 C_{J} r_{k+n}=\left|x-x_{k+n}\right| \leq|x-y|+\left|y-x_{k+n}\right| \leq|x-y|+r_{k+n}, \quad n=0, \ldots, p .
$$

Therefore, we have

$$
\left(4 C_{J}-1\right) r_{k+n} \leq\left(4 C_{J}+1\right) r_{k+m}, \quad m, n=0, \ldots, p .
$$

Since no center of a disk belongs to any other disk, it is then easy to show that, for $m \neq n$,

$$
\left|x_{k+n}-x_{k+m}\right| \geq\left(4 C_{J}-1\right) /\left(4 C_{J}+1\right) r_{k},
$$

which provides a uniform bound on $p$ since only a finite number of such $x_{i}$ can satisfy (3.4) while being within $r_{i}$ of the common point $y$.

The second statement now follows easily by using the bound on the ratio of the radii of the two circles and elementary geometry.

We next consider a circle, centered at $x$, which covers all of $\Omega_{i}$. We decompose this circle into annuli with a fixed ratio between the outer and inner circles of any annulus. It is easy to see that the number of $x_{i}$ contained in each annulus will be uniformly bounded since the radii of the disks associated with an annulus are determined by the distance to $x$. This shows that $r_{i} \rightarrow 0$ and also that we can, by a simple covering argument, bound the number of $x_{i}$ 's that are at a distance larger than $r$ from $x$ by $M \log (H / r)$. 
Proof of Lemma 3.2. We will now confine $x$ to be a centroid of an element $K$; by following [6, Formula (4.9.6)] we can then easily obtain our estimate for any $x \in K$. We denote by $N=N(h)$ the index of the first $B_{i}$ which lies entirely inside $K$.

We will work with averages $\bar{u}_{B_{i}}$ of sufficiently smooth functions over the circular disks. We note that the average of a linear function over a circular disk $B_{i}$ coincides with the value at its center if $B_{i} \subset K$.

Then, by following [14] and using the triangle inequality, we find that

$$
\begin{array}{r}
\left|u\left(x_{N}\right)-\bar{u}_{B_{0}}\right| \leq \sum_{i=0}^{N-1}\left|\bar{u}_{B_{i}}-\bar{u}_{B_{i+1}}\right| \\
\leq \sum_{i=0}^{N-1}\left(\left|\bar{u}_{B_{i}}-\bar{u}_{B_{i} \cap B_{i+1}}\right|+\left|\bar{u}_{B_{i} \cap B_{i+1}}-\bar{u}_{B_{i+1}}\right|\right) .
\end{array}
$$

The expression $\bar{u}_{B_{i}}-\bar{u}_{B_{i} \cap B_{i+1}}$ is invariant under a shift of $u$ by a constant. By replacing $u$ by $u-\bar{u}_{\mathcal{B}_{i}}$, we therefore find that

$$
\begin{array}{r}
\left|\bar{u}_{B_{i}}-\bar{u}_{B_{i} \cap B_{i+1}}\right| \leq \frac{1}{\left|B_{i} \cap B_{i+1}\right|} \int_{B_{i} \cap B_{i+1}}\left|u-\bar{u}_{\mathcal{B}_{i}}\right| \\
\leq \frac{1}{\left|B_{i} \cap B_{i+1}\right|} \int_{B_{i}}\left|u-\bar{u}_{\mathcal{B}_{i}}\right|=\frac{\left|B_{i}\right|}{\left|B_{i} \cap B_{i+1}\right|} f_{B_{i}}\left|u-\bar{u}_{B_{i}}\right| .
\end{array}
$$

Here $f_{B_{i}} v$ denotes the average of $v$ over the disk $B_{i}$. The second term of (3.5) can be estimated similarly, and we can now use the second statement of Lemma 3.3 to obtain

$$
\left|u\left(x_{N}\right)-\bar{u}_{B_{0}}\right| \leq(2 M) \sum_{i=0}^{N-1} f_{B_{i}}\left|u-\bar{u}_{B_{i}}\right| .
$$

By using Lemma 2.3 for the disks $B_{i}$, we obtain

$$
f_{B_{i}}\left|u-\bar{u}_{B_{i}}\right| \leq C \int_{B_{i}} \frac{|\nabla u(z)|}{r_{i}} d z
$$

We now use the Cauchy-Schwarz inequality and obtain

$$
\left|u\left(x_{N}\right)-\bar{u}_{B_{0}}\right|^{2} \leq C N \sum_{i=0}^{N-1} \int_{B_{i}}|\nabla u(z)|^{2} d z .
$$

By using the finite covering property - the first statement of Lemma 3.3 - this sum can be estimated by $\int_{\Omega_{i}}|\nabla u(z)|^{2} d z$.

Given that $B_{N}$ is the first disk which is a subset of $K$ and that $x_{N}$ lies on the boundary of $B_{N-1}$, we find that $\operatorname{dist}\left(x_{N}, \partial K\right) \leq 2 r_{N-1}$. We also know that

$$
r_{N-1} \leq \frac{4 C_{J}+1}{4 C_{J}-1} r_{N} \text { and that } r_{N}=\frac{\left|x-x_{N}\right|}{4 C_{J}} .
$$

Since

$$
\operatorname{dist}(x, \partial K) \leq\left|x_{N}-x\right|+\operatorname{dist}\left(x_{N}, \partial K\right)
$$

Copyright $@$ by SIAM. Unauthorized reproduction of this article is prohibited. 
we see that $\left|x-x_{N}\right|$ is bounded from below by $c\left(C_{J}\right) h$, where $c\left(C_{J}\right)>0$ and $h$ is the diameter of the element $K$. We can now use the logarithmic estimate of $N$ - the fourth statement of Lemma 3.3-and find that

$$
\left|u\left(x_{N}\right)-\bar{u}_{B_{0}}\right|^{2} \leq C(1+\log (H / h)) \int_{\Omega_{i}}|\nabla u(z)|^{2} d z .
$$

It is also elementary to estimate $\left|\bar{u}_{B_{0}}-\bar{u}_{\Omega_{i}}\right|$. We find that

$$
\left|\bar{u}_{B_{0}}-\bar{u}_{\Omega_{i}}\right| \leq\left|B_{0}\right|^{-1}\left\|u-\bar{u}_{\Omega_{i}}\right\|_{L_{1}\left(B_{0}\right)} \leq\left|B_{0}\right|^{-1}\left\|u-\bar{u}_{\Omega_{i}}\right\|_{L_{1}\left(\Omega_{i}\right)} .
$$

We complete the argument by using the Cauchy-Schwarz inequality, Lemma 2.2, and the fact that there is a bound on $\left|\Omega_{i}\right|$ in terms of $\left|B_{0}\right|$. We note that this term does not require any logarithmic factor.

We then have a bound for one point in the element $K$, and, as already indicated, we can easily estimate the difference between the values at any pair of points in the element $K$ as in [6, Formula (4.9.6)].

A second important tool provides estimates of the edge functions.

LEMma 3.4. The edge function $\theta_{\mathcal{E}^{i k}}$ can be bounded as follows:

$$
\left\|\theta_{\mathcal{E}^{i k}}\right\|_{H^{1}\left(\Omega_{i}\right)}^{2} \leq C\left(1+\log \left(H_{i} / h_{i}\right)\right),
$$

and

$$
\left\|\theta_{\mathcal{E}^{i k}}\right\|_{L_{2}\left(\Omega_{i}\right)}^{2} \leq C H_{i}^{2}\left(1+\log \left(H_{i} / h_{i}\right)\right) .
$$

Here the constant $C$ depends only on the John parameter $C_{J}\left(\Omega_{i}\right)$. We can remove the logarithmic factor from (3.7) if all angles in the triangulation are acute, i.e.,

$$
\left\|\theta_{\mathcal{E}^{i k}}\right\|_{L_{2}\left(\Omega_{i}\right)}^{2} \leq C H_{i}^{2} .
$$

A proof of this lemma is given in [16].

4. Proof of Theorem 3.1. As in many other proofs of results on domain decomposition algorithms, we will work with one subdomain $\Omega_{i}$ at a time. With local bounds, there are now no difficulties in handling variations of the coefficients across the interface.

We recall that the coarse space is spanned by the $\theta_{\mathcal{V}}$, the discrete harmonic extensions of the restrictions of the standard nodal basis functions to $\Gamma$, and the edge functions $\theta_{\mathcal{E}^{i k}}$. The coarse space component $u_{0} \in V_{0}$ in the decomposition of an arbitrary finite element function $u(x)$ can be chosen as

$$
u_{0}(x)=\sum_{\ell} u\left(\mathcal{V}^{\ell}\right) \theta_{\mathcal{V}^{\ell}}(x)+\sum_{i k} \bar{u}_{\mathcal{E}^{i k}} \theta_{\mathcal{E}^{i k}}(x)
$$

Here $\bar{u}_{\mathcal{E}^{i k}}$ is the average of $u$ over the edge. This interpolation formula is a twodimensional analog of [28, Formula (5.13)].

Since the operator defined by (4.1) reproduces constants, we can replace $u$ by $u-\bar{u}_{\Omega_{i}}$ and $u_{0}$ by $u_{0}-\bar{u}_{\Omega_{i}}$. We now estimate the terms of the right-hand side of the resulting formula term by term. The energy of the vertex basis functions is uniformly bounded, and the values of $u\left(\mathcal{V}^{\ell}\right)-\bar{u}_{\Omega_{i}}$ are bounded by using Lemma 3.2. Turning to the edge terms, we use Lemma 3.4 to estimate the energy of the edge basis functions. The coefficient $\bar{u}_{\mathcal{E}^{i k}}-\bar{u}_{\Omega_{i}}$ is bounded by $\left\|u-\bar{u}_{\Omega_{i}}\right\|_{L_{\infty}\left(\Omega_{i}\right)}$. Thus,

$$
\left|u_{0}\right|_{H^{1}\left(\Omega_{i}\right)}^{2}=\left|u_{0}-\bar{u}_{\Omega_{i}}\right|_{H^{1}\left(\Omega_{i}\right)}^{2} \leq C(1+\log (H / h))^{2}|u|_{H^{1}\left(\Omega_{i}\right)}^{2},
$$


and we find that

$$
a\left(u_{0}, u_{0}\right) \leq C(1+\log (H / h))^{2} a(u, u) .
$$

Remark 1. If the subdomains $\Omega_{i}$ have Lipschitz boundaries, we can estimate the edge averages by using the Cauchy-Schwarz inequality and an elementary trace theorem; see Nečas [23, Theorem 1.2]. This eliminates one of the two logarithmic factors.

Similarly, we can prove that

$$
\left\|u-u_{0}\right\|_{L_{2}\left(\Omega_{i}\right)}^{2} \leq C(1+\log (H / h))^{2} H_{i}^{2}|u|_{H^{1}\left(\Omega_{i}\right)}^{2} .
$$

If all angles of the triangulation of $\Omega_{i}$ are acute, then by using (3.8) of Lemma 3.4 we can remove one of the logarithmic factors, and if the subdomain boundaries are Lipschitz, no logarithmic factors are required on the right-hand side of (4.3); see [28, Lemma 4.25] for a three-dimensional result for regular subdomains.

Remark 2. In the case of linear elasticity, we must include the restriction of all rigid body modes to the edges in the coarse space; see [19] or [27] for a discussion of the necessity of this null space property. In this case, we should extend the values given on the interface into the subdomains by solving homogeneous discrete elasticity problems.

We now turn to the estimate related to the local spaces. Again, we will carry out the work on one subdomain $\Omega_{i}$ at a time. Let $w:=u-u_{0}$, and define a local term in the decomposition by $u_{j}=I^{h}\left(\theta_{j} w\right)$. We can borrow extensively from [28, sections 3.2 and 3.6]. Thus, $I^{h}$ interpolates into $V^{h}$, and the $\theta_{j} \in R_{j}^{T} V_{j}$ provide a partition of unity. These functions vary between 0 and 1 , and their gradients are bounded by $\left|\nabla \theta_{j}\right| \leq C / \delta_{j}$ and vanish outside the regions of overlap $\Omega_{j, \delta_{j}}$.

We note that the number of $\Omega_{j}^{\prime}$ that intersect $\Omega_{i}$ is uniformly bounded; we will consider only the contribution from one of them, $\Omega_{j}^{\prime}$. As in our earlier work [28, section 3.5], the only term that requires a careful consideration is $\nabla \theta_{j} w$. We cover the set $\Omega_{j, \delta_{j}} \cap \Omega_{i}$ by square patches with sides on the order of $\delta_{j}$ and note that on the order of $H_{i} / \delta_{j}$ of them will suffice. Just as in the proof of [28, Lemma 3.10], we have

$$
\int_{\Omega_{i}}\left|\nabla \theta_{j} w\right|^{2} \leq C / \delta_{j}^{2}\left(\delta_{j}^{2}|w|_{H^{1}\left(\Omega_{i}\right)}^{2}+\left(H_{i} / \delta_{j}\right) \delta_{j}^{2}\|w\|_{H^{1}\left(\Omega_{i}\right)}^{2}\right) .
$$

The proof is completed by combining this inequality with the bounds in (4.2) and (4.3).

Remark 3. Should the overlap between the subdomains be more generous in places, we can modify the relevant $\theta_{j}$ by making them vary from 0 to 1 over a distance of $\delta_{j}$, effectively making the set $\Omega_{j, \delta_{j}}$ more evenly wide.

5. Numerical examples. Numerical examples are presented in this section for the three different types of subdomains shown in Figure 5.1. Type 1 subdomains have a square geometry and are partitioned into square bilinear elements. Type 2 subdomains also consist of square bilinear elements, but their boundaries have a ragged shape. For type 3 subdomains, we employ equilateral linear triangular elements, and the edges of the subdomains have both straight-line and fractal segments. Numerical examples are also presented for subdomains obtained from a mesh partitioner; see Figure 5.5.

The main purpose of this first set of numerical examples is to verify certain estimates used in the proof of Theorem 3.1. For type 1 and 2 subdomains, the ratio 

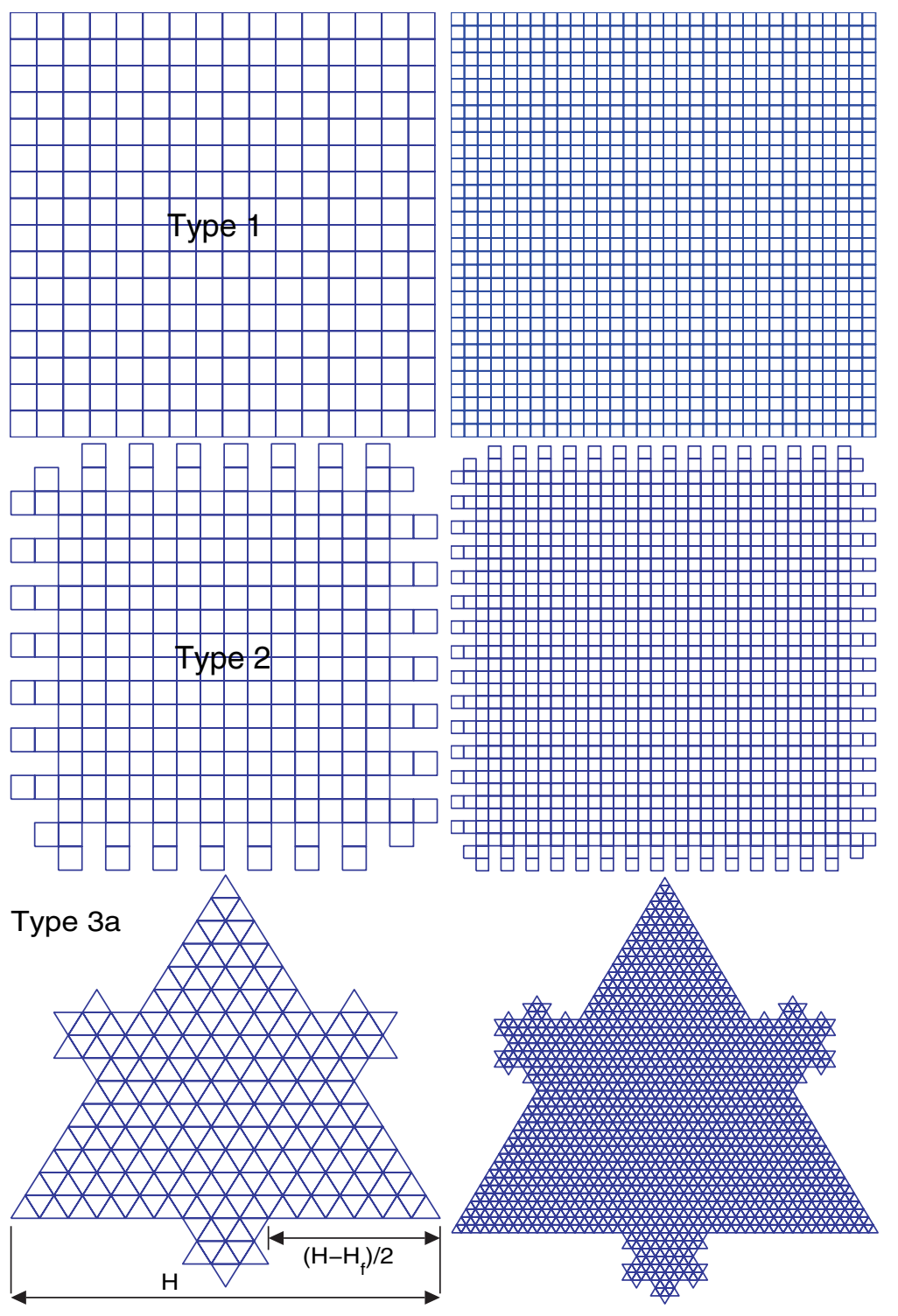

FIG. 5.1. Three types of subdomains used in the numerical examples. Two levels of mesh refinement are shown, and the type 3 subdomains have a constant value of $H / H_{f}=5$. For each refinement of type 3 subdomains, every element edge on the fractal part of the boundary is first divided into three shorter edges of $1 / 3$ the length. The middle one of these edges is then replaced by two other edges with which it forms an equilateral triangle. Thus, for fixed $\mathrm{H} / \mathrm{H}_{f}$, the length of the fractal part of the boundary increases by a factor of $4 / 3$ with each additional level of refinement.

$H / h$ is increased by a factor of 2 with each additional level of mesh refinement. At the $i$ th $(i \geq 0)$ level of refinement for type 3 subdomains,

$$
H / h=\left(H / H_{f}\right) 3^{i+1},
$$

where $H / H_{f}=5$ is fixed for type 3 a subdomains and $H / H_{f}=5+2 i$ for type $3 \mathrm{~b}$. We note that in the asymptotic limit the fractal segment lengths grow by a factor of $4 / 3$ with each mesh refinement for both type 3 subdomains, whereas the straight-line 

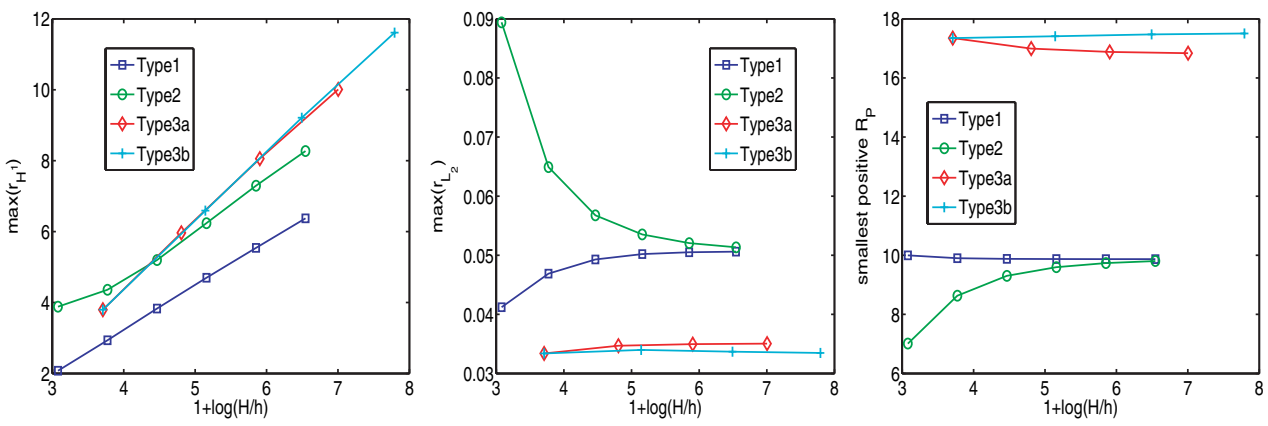

FIG. 5.2. Rayleigh quotients for different subdomain types.

segment lengths remain bounded. The primary difference between type $3 \mathrm{a}$ and $3 \mathrm{~b}$ subdomains is that $H / H_{f}$ is fixed in the former while $H / H_{f}$ increases without bound in the latter. Thus, for type $3 \mathrm{~b}$ subdomains, the protruding regions bounded by fractal segments decrease in area, while fractal segment lengths continue to increase with mesh refinement.

We recall the estimates (4.2) and (4.3) from the proof of Theorem 3.1 and consider the associated Rayleigh quotients

$$
r_{H^{1}}=\frac{\left|u_{0}\right|_{H^{1}\left(\Omega_{i}\right)}^{2}}{|u|_{H^{1}\left(\Omega_{i}\right)}^{2}} \quad \text { and } \quad r_{L_{2}}=\frac{\left\|u-u_{0}\right\|_{L_{2}\left(\Omega_{i}\right)}^{2}}{|u|_{H^{1}\left(\Omega_{i}\right)}^{2}}
$$

and also the Rayleigh quotient

$$
r_{P}=\frac{|u|_{H^{1}\left(\Omega_{i}\right)}^{2} .}{\|u\|_{L_{2}\left(\Omega_{i}\right)}^{2}}
$$

Stationary values of these Rayleigh quotients correspond to eigenvalues of generalized eigenproblems. For example, a stationary value of $r_{P}$ is an eigenvalue $\lambda$ of

$$
K \phi=\lambda M \phi,
$$

where $K$ and $M$ are the stiffness and mass matrices, respectively, of the finite element discretization of $\Omega_{i}$ with Neumann boundary conditions. We note in this case that the reciprocal of the smallest positive eigenvalue provides an estimate of the constant in Lemma 2.2. Maximum values of $r_{H^{1}}$ and $r_{L_{2}}$, along with the smallest positive stationary value of $r_{P}$, are plotted versus $1+\log (H / h)$ in Figure 5.2. We observe essentially the same dependence on $\log (H / h)$ for all four subdomain types. The maximum value of $r_{H^{1}}$ appears to vary linearly with $\log (H / h)$ in the asymptotic limit, whereas the maximum value of $r_{L_{2}}$ appears to be converging to a constant. This behavior is consistent with (4.2) and (4.3), but the estimates do not appear to be sharp for the different subdomain types and ranges of $H / h$ considered. Consistent with the isoperimetric inequality, the rightmost plot in Figure 5.2 suggests that the smallest positive stationary value of $r_{P}$ is uniformly bounded from below. We have yet to identify a subdomain shape with numerical behavior essentially different from the regular-shaped case.

The next group of numerical examples are for the domain decompositions shown in Figure 5.3. For type 3 subdomains, we fix $H / H_{f}=5$. Homogeneous essential 

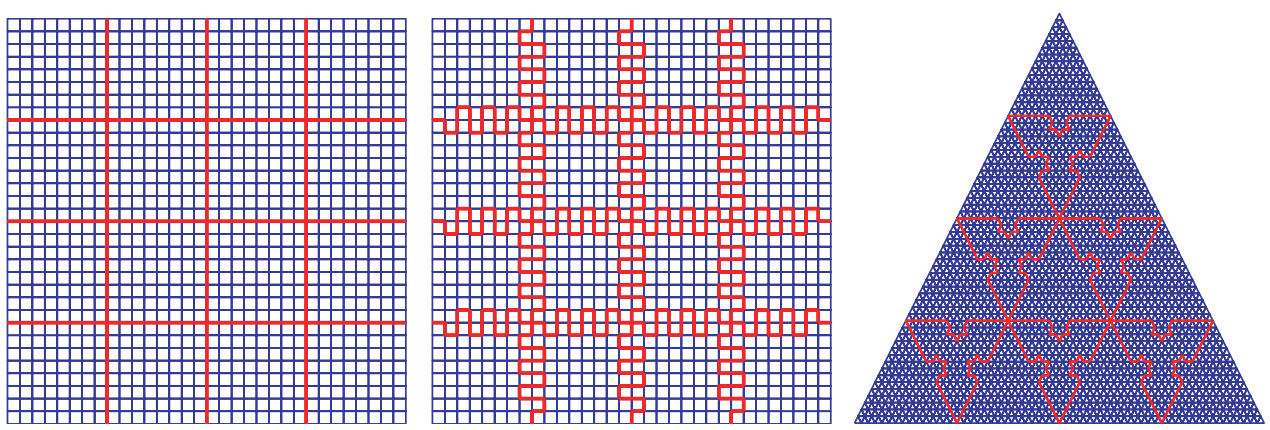

FIG. 5.3. Sample domain decompositions, corresponding to subdomains of type 1, 2, and 3a, used in numerical examples. Subdomain boundaries are shown in red.

TABLE 5.1

Results for domain decompositions shown in Figure 5.3. Table headings iter, cond, and ndof denote the number of iterations, condition number estimates, and the number of unknowns in the problem, respectively. Material properties are the same for all subdomains.

\begin{tabular}{|c|c|c|c|c|c|}
\hline Type & $H / h$ & $d_{j}$ & iter & cond & ndof \\
\hline 1 & 8 & 1 & 25 & 9.11 & 1056 \\
& 16 & 3 & 29 & 11.07 & 4160 \\
& 32 & 7 & 31 & 12.99 & 16,512 \\
& 64 & 15 & 32 & 14.87 & 65,792 \\
& 128 & 31 & 34 & 16.71 & 262,656 \\
\hline 2 & 8 & 1 & 28 & 13.53 & 1056 \\
& 16 & 3 & 30 & 15.70 & 4160 \\
& 32 & 7 & 32 & 17.81 & 16,512 \\
& 64 & 15 & 33 & 19.76 & 65,792 \\
& 128 & 31 & 34 & 21.60 & 262,656 \\
\hline 3 & 15 & 3 & 30 & 18.56 & 1830 \\
& 45 & 11 & 34 & 22.79 & 16,290 \\
& 135 & 35 & 36 & 26.66 & 146,070 \\
& 405 & 107 & 37 & 30.14 & $1,313,010$ \\
\hline
\end{tabular}

boundary conditions are applied to the bottom edge of each domain, and $\rho_{i}=1$ for each subdomain. Preconditioned conjugate gradients are used to solve the associated linear systems to a relative residual tolerance of $10^{-8}$ for random right-hand sides. The amount of overlap is specified by the integer $d_{j}$. If $\Omega_{i, h}$ contains all of the nodes in the closure of $\Omega_{i}$, then the nodes in overlapping subdomain $\Omega_{i}^{\prime}$ are the union of $\Omega_{i, h}$ with all nodes a graph distance $d_{j}$ or less from one or more nodes in $\Omega_{i, h}$. We note that nodes on the boundary of subdomain $\Omega_{i}$ are also contained in $\Omega_{i, h}$; the designation nonoverlapping refers to an element rather than to a nodal decomposition.

The numbers of iterations and condition number estimates from conjugate gradient iterations are reported in Table 5.1. The condition numbers from Table 5.1 are also plotted in Figure 5.4. Provided the overlap $d_{j}+1$ remains in a fixed proportion to $H / h$, i.e., $H / \delta$ is constant, the growth in condition number appears to be bounded by $C(1+\log (H / h))$ for all three subdomain types considered. These results are consistent with Theorem 3.1 but suggest that the estimate in the theorem may not be sharp.

The next group of examples is identical to the previous one, but we now set $\rho_{i}=1$ for the bottom two rows of subdomains and $\rho_{i}=\sigma$ for the top two rows 


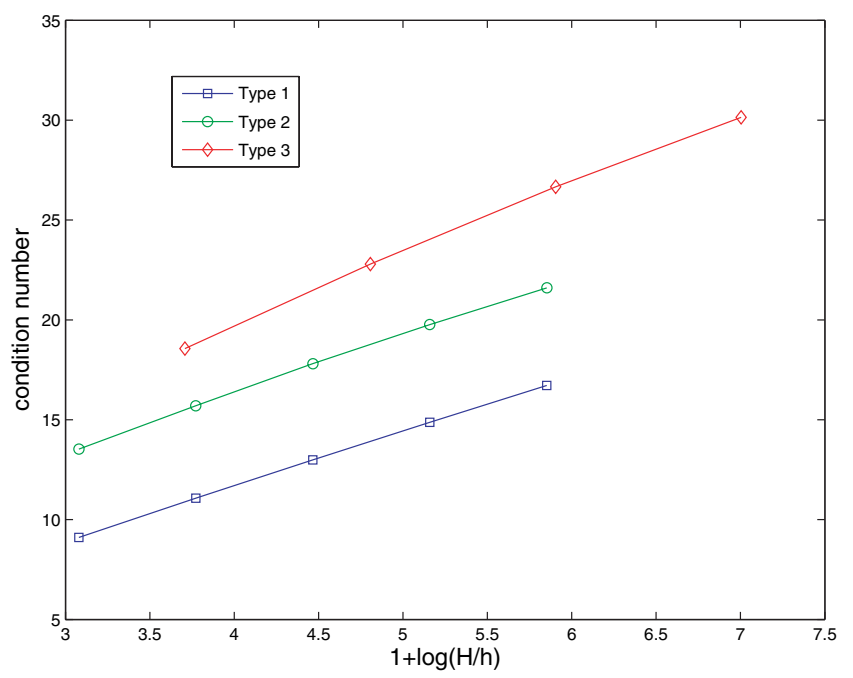

FIG. 5.4. Condition numbers versus $1+\log (H / h)$ for three different subdomain types.

TABLE 5.2

Results for domain decompositions shown in Figure 5.3. The bottom two rows of subdomains have $\rho_{i}=1$, and the top two rows have $\rho_{i}=\sigma$. Material property jumps are aligned with subdomain boundaries.

\begin{tabular}{|c|c|c|c|c|c|c|}
\hline Type & $H / h$ & $d_{j}$ & $\sigma$ & iter & cond & ndof \\
\hline 1 & 16 & 3 & $10^{-4}$ & 32 & 10.10 & 4160 \\
& & & $10^{-2}$ & 29 & 10.16 & \\
& & & 1 & 29 & 11.07 & \\
& & & $10^{2}$ & 30 & 11.45 & \\
\hline 2 & 16 & 3 & $10^{4}$ & 32 & 11.45 & \\
& & & $10^{-2}$ & 33 & 11.86 & 4160 \\
& & & 1 & 30 & 12.19 & \\
& & & $10^{2}$ & 31 & 16.75 & \\
\hline 3 & 45 & 11 & $10^{4}$ & 32 & 16.76 & \\
& & & $10^{-2}$ & 34 & 18.84 & 16,290 \\
& & & 1 & 34 & 22.79 & \\
& & & $10^{2}$ & 33 & 27.72 & \\
& & & $10^{4}$ & 35 & 27.86 & \\
\hline
\end{tabular}

of subdomains in Figure 5.3. Consistent with the theory, the results in Table 5.2 suggest that condition numbers are bounded independently of material property jumps between subdomains.

The final group of examples deals with subdomains generated by a mesh partitioner. Consider a graph in which each vertex of the graph corresponds to a finite element. There is an edge between two vertices in the graph if the corresponding finite elements share an edge, i.e., they have two nodes in common. The graph partitioning software Metis [15] is then used to decompose such graphs into $N$ subdomains. Example domain decompositions obtained in this manner are shown in Figure 5.5 for the same square geometry as in the previous examples. Results in Table 5.3 suggest that using a general-purpose mesh partitioner to generate subdomains does not degrade performance significantly. Results are also shown in the table for problems in which 

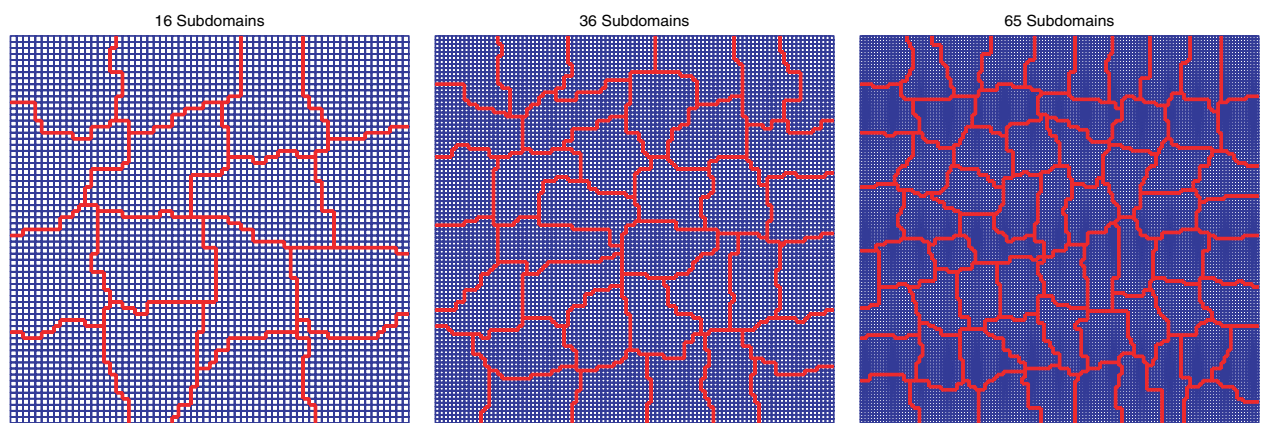

FIG. 5.5. Sample domain decompositions obtained from a mesh partitioner.

TABLE 5.3

Results for square subdomains and subdomains obtained from a mesh partitioner, where $N$ designates the number of subdomains. In the upper right quadrant of the square domain $\rho(x)=\sigma$, and elsewhere $\rho(x)=1$. For $N=64$ and $H / h=16$, the mesh partitioner generated one subdomain consisting of two disconnected components. These two components were treated as distinct subdomains resulting in $N=65$. Results in the bottom part of the table are for a checkerboard arrangement of material properties.

\begin{tabular}{|c|c|c|c|c|c|c|c|c|}
\hline \multirow[b]{2}{*}{$N$} & \multirow[b]{2}{*}{$H / h$} & \multirow[b]{2}{*}{$\sigma$} & \multirow[b]{2}{*}{$d_{j}$} & \multicolumn{2}{|c|}{$\begin{array}{c}\text { Square } \\
\text { subdomains }\end{array}$} & \multicolumn{2}{|c|}{$\begin{array}{c}\text { Mesh } \\
\text { partitioner }\end{array}$} & \multirow[b]{2}{*}{ ndof } \\
\hline & & & & iter & cond & iter & cond & \\
\hline \multirow[t]{2}{*}{16} & 16 & 1 & 3 & 29 & 11.07 & 31 & 10.31 & 4160 \\
\hline & 32 & & 7 & 31 & 12.99 & 33 & 11.96 & 16,512 \\
\hline \multirow[t]{2}{*}{36} & 16 & & 3 & 31 & 11.87 & 36 & 14.05 & 9312 \\
\hline & 32 & & 7 & 33 & 14.11 & 39 & 14.78 & 37,056 \\
\hline $64 / 65$ & 16 & & 3 & 33 & 12.25 & 38 & 14.49 & 16,512 \\
\hline 64 & 32 & & 7 & 36 & 14.63 & 41 & 15.42 & 65,792 \\
\hline \multirow[t]{5}{*}{25} & \multirow[t]{5}{*}{16} & $10^{-4}$ & 3 & 31 & 11.55 & 38 & 17.18 & \multirow[t]{5}{*}{6480} \\
\hline & & $10^{-2}$ & & 31 & 11.55 & 38 & 17.18 & \\
\hline & & 1 & & 30 & 11.56 & 37 & 17.15 & \\
\hline & & $10^{2}$ & & 34 & 14.34 & 38 & 17.14 & \\
\hline & & $10^{4}$ & & 34 & 14.58 & 38 & 17.14 & \\
\hline \multicolumn{9}{|c|}{$5 \times 5$ checkerboard with $(16 \cdot 5)^{2}$ total elements } \\
\hline \multirow[t]{3}{*}{20} & & $10^{-4}$ & 3 & & & 34 & 28.39 & \multirow[t]{3}{*}{6480} \\
\hline & & 1 & & & & 36 & 14.39 & \\
\hline & & $10^{4}$ & & & & 41 & 32.53 & \\
\hline \multirow[t]{3}{*}{25} & & $10^{-4}$ & 3 & 26 & 9.40 & 46 & 46.87 & \multirow[t]{3}{*}{6480} \\
\hline & & 1 & & 30 & 11.56 & 37 & 17.15 & \\
\hline & & $10^{4}$ & & 26 & 9.55 & 37 & 18.86 & \\
\hline \multirow[t]{3}{*}{30} & & $10^{-4}$ & 3 & & & 36 & 16.69 & \multirow[t]{3}{*}{6480} \\
\hline & & 1 & & & & 34 & 13.09 & \\
\hline & & $10^{4}$ & & & & 43 & 24.44 & \\
\hline
\end{tabular}

$\rho(x)=\sigma$ in the upper right quadrant of the square domain and $\rho(x)=1$ elsewhere. The bottom part of Table 5.3 shows results for a $5 \times 5$ checkerboard where $\rho(x)=1$ in red squares and $\rho(x)=\sigma$ in black squares. Except for the checkerboard problem decomposed into 25 square subdomains, the subdomain boundaries are not aligned with jumps in material properties. Although our theory does not apply to such cases, good performance is evident for both regular and irregular domain decompositions.

Acknowledgments. The authors are grateful to Professor Fanghua Lin of the Courant Institute for introducing the third author to John domains and Poincaré's 
inequality for very general domains. They are also grateful to Professors Piotr Hajłasz and Marcus Sarkis for their interest and insight.

\section{REFERENCES}

[1] G. Acosta, R. G. Durán, And M. A. Muschietti, Solutions of the divergence operator on John domains, Adv. Math., 206 (2006), pp. 373-401.

[2] B. Bojarski, Remarks on Sobolev imbedding inequalities, in Complex Analysis, Joensuu 1987, Lecture Notes in Math. 1351, Springer-Verlag, Berlin, 1988, pp. 52-68.

[3] J. Bramble, A second order finite difference analog of the first biharmonic boundary value problem, Numer. Math., 9 (1966), pp. 236-249.

[4] J. H. Bramble, J. E. Pasciak, and A. H. Schatz, The construction of preconditioners for elliptic problems by substructuring. I, Math. Comp., 47 (1986), pp. 103-134.

[5] S. Brenner and L.-Y. Sung, Discrete Sobolev and Poincaré inequalities via Fourier series, East-West J. Numer. Math., 8 (2000), pp. 83-92.

[6] S. C. Brenner And L. R. Scott, The Mathematical Theory of Finite Element Methods, Texts Appl. Math. 15, 2nd ed., Springer-Verlag, New York, 2002.

[7] S. M. Buckley and P. Koskela, Sobolev-Poincaré implies John, Math. Res. Lett., 2 (1995), pp. $577-593$.

[8] C. R. Dohrmann, A. Klawonn, and O. B. Widlund, A family of energy minimizing coarse spaces for overlapping Schwarz preconditioners, in Proceedings of the 17th International Conference on Domain Decomposition Methods in Science and Engineering, Strobl, Austria, 2006, U. Langer, M. Discacciati, D. Keyes, O. Widlund, and W. Zulehner, eds., Lect. Notes Comput. Sci. Eng. 60, Springer-Verlag, Berlin, 2007.

[9] C. R. Dohrmann and O. B. Widlund, An Overlapping Schwarz Algorithm for Almost Incompressible Elasticity, manuscript, 2007.

[10] M. Dryja, M. V. Sarkis, And O. B. Widlund, Multilevel Schwarz methods for elliptic problems with discontinuous coefficients in three dimensions, Numer. Math., 72 (1996), pp. 313348.

[11] M. Dryja, B. F. Smith, And O. B. Widlund, Schwarz analysis of iterative substructuring algorithms for elliptic problems in three dimensions, SIAM J. Numer. Anal., 31 (1994), pp. 1662-1694.

[12] H. Federer and W. H. Fleming, Normal and integral currents, Ann. of Math. 2, 72 (1960), pp. $458-520$.

[13] I. G. Graham, P. Lechner, and R. Scheichl, Domain decomposition for multiscale PDEs, Numer. Math., (2007), pp. 589-626.

[14] P. HAJŁASZ, Sobolev inequalities, truncation method, and John domains, in Papers on Analysis, Rep. Univ. Jyväskylä Dep. Math. Stat. 83, University of Jyväskylä, Jyväskylä, 2001, pp. 109-126.

[15] G. Karypis and V. Kumar, METIS Version 4.0, University of Minnesota, Department of Computer Science, Minneapolis, MN, 1998.

[16] A. Klawonn, O. Rheinbach, and O. B. Widlund, An analysis of a FETI-DP algorithm on irregular subdomains in the plane, SIAM J. Numer Anal., to appear.

[17] A. Klawonn and O. B. Widlund, Dual-primal FETI methods for linear elasticity, Comm. Pure Appl. Math., 59 (2006), pp. 1523-1572.

[18] F. Lin And X. Yang, Geometric Measure Theory-An Introduction, Adv. Math. (Beijing/Boston) 1, Science Press, Beijing, 2002.

[19] J. MANDEL, Iterative solvers by substructuring for the p-version finite element method, Comput. Methods Appl. Mech. Engrg., 80 (1990), pp. 117-128.

[20] V. G. MAZ'JA, Classes of domains and imbedding theorems for function spaces, Soviet Math. Dokl., 1 (1960), pp. 882-885.

[21] V. G. MaZ'JA, Sobolev Spaces, Springer Ser. Soviet Math., Springer-Verlag, Berlin, 1985, translated from the Russian by T. O. Shaposhnikova.

[22] V. G. MaZ'ya And S. V. Poborchi, Differentiable Functions on Bad Domains, World Scientific, River Edge, NJ, 1997.

[23] J. NEČAs, Les méthodes Directes en Théorie des Équations Elliptiques, Academia, Prague, 1967.

[24] M. SARKIS, Nonstandard coarse spaces and Schwarz methods for elliptic problems with discontinuous coefficients using non-conforming elements, Numer. Math., 77 (1997), pp. 383-406.

[25] M. V. SARKIS, Partition of unity coarse spaces: Enhanced versions, discontinuous coefficients and applications to elasticity, in Domain Decomposition Methods in Science and Engineer-

Copyright $@$ by SIAM. Unauthorized reproduction of this article is prohibited. 
ing, I. Herrera, D. E. Keyes, O. B. Widlund, and R. Yates, eds., Natl. Auton. Univ. Mex., México, 2003, pp. 149-158.

[26] R. Scheichl and E. Vainikko, Additive Schwarz and aggregation-based coarsening for elliptic problems with highly variable coefficients, Computing, 80 (2007), pp. 319-343.

[27] B. F. Smith, P. Buørstad, and W. Gropp, Domain Decomposition: Parallel Multilevel Methods for Elliptic Partial Differential Equations, Cambridge University Press, New York, 1996.

[28] A. Toselli And O. B. Widuund, Domain Decomposition Methods - Algorithms and Theory, Springer Ser. Comput. Math. 34, Springer-Verlag, Berlin, 2005.

Copyright (c) by SIAM. Unauthorized reproduction of this article is prohibited. 